\title{
Construction of Secure Elliptic Cryptosystems Using CM Tests and Liftings
}

\author{
Jinhui Chao $^{1}$, Osamu Nakamura ${ }^{2}$, Kohji Sobataka $^{1}$, and Shigeo Tsujii ${ }^{2}$ \\ 1 Dept. of Electrical and Electronic Engineering, Chuo University, Tokyo, Japan \\ 2 Dept. of Information and Engineering Systems, Chuo University, Tokyo, Japan \\ jchao@elect.chuo-u.ac.jp, soba@chao.elect.chuo-u.ac.jp, \\ tsujii@ise.chuo-u.ac.jp
}

\begin{abstract}
Elliptic curves over number fields with CM can be used to design non-isogenous elliptic cryptosystems over finite fields efficiently. The existing algorithm to build such CM curves, so-called the CM field algorithm, is based on analytic expansion of modular functions, costing computations of $O\left(2^{5 h / 2} h^{21 / 4}\right)$ where $h$ is the class number of the endomorphism ring of the CM curve. Thus it is effective only in the small class number cases.

This paper presents polynomial time algorithms in $h$ to build CM elliptic curves over number fields. In the first part, probabilistic probabilistic algorithms of CM tests are presented to find elliptic curves with $\mathrm{CM}$ without restriction on class numbers. In the second part, we show how to construct ring class fields from ray class fields. Finally, a deterministic algorithm for lifting the ring class equations from small finite fields thus construct CM curves is presented. Its complexity is shown as $O\left(h^{7}\right)$.
\end{abstract}

\section{Introduction}

Elliptic curves over finite fields have been used in recent public key cryptosystems, authentication and signature schemes. The discrete logarithm problems over the elliptic curves can resist all known subexponential attacks, which then can implement cryptographic schemes in higher speed and less key sizes while retain the same security comparing with traditional cryptographic functions [14] 22] 21].

Among the methods to construct explicitly secure elliptic curves over finite fields for cryptosystem applications, the point-counting algorithms, now known as the SEA algorithms can find secure curves over finite fields from randomly selected elliptic curves, but still be quite time consuming since they generally need to be repeated many times until a secure curve is found [27] 28] [8] 24] 20] [10]. Another difficulty of this approach is that when one wishes to choose different curves for different users or periodically change curves over finite fields in the same cryptosystem, he has to undergo the whole process of the above calculations, or it always takes the same computations in order to obtain any new secure curves and cryptosystems. 
According to [16, if the same curve over a finite field is repeatedly used in an elliptic cryptosystem, even each time with a random base point, one can easily transform the new discrete logarithm problem into the old one. Thus the old database can be made good use of such that one can attack this kind of cryptosystems faster, comparing with those which switch each time to a new or non-isogenous curve over a finite field. Furthermore, if one is willing to build a large database, then with certain variations of the Baby step Giant step algorithm, one can attack the cryptosystems using a fixed curve over a finite field in time of $O\left(q^{1 / d}\right), d>2$, rather than the standard complexity of the Baby step and Giant step algorithm: $O(\sqrt{q})$.

Another approach to build secure elliptic curves over finite fields, which is much faster and meets the requirement to change to non-isogenous curves frequently, is to use a family of elliptic curves defined over number fields, i.e. those with complex multiplication or CM elliptic curves [23] 2] 3] [4] 19].

In fact, the CM curves may not be easy to find, but once a CM curve over a number field is built, one can use very simple and fast algorithms to design directly non-isogenous curves over finite fields with different and maybe prespecified (almost prime) orders, therefore different secure cryptosystems as many as one wishes, if he changes the characteristics and extension degrees of the finite definition fields. This can be done by efficient algorithms of reduced quadratic forms (see appendix) or the Cornacchia algorithm.

As to the security of using CM curves, it is known that all elliptic curves over finite fields are with CM (so usually they are not referred to as CM curves and we will leave the name exclusively to curves over number fields). Furthermore, each elliptic curve over a finite field is the reduction of an CM curve (known as its Deuring's lifting) over a certain number field. If there were any attack which works effective particularly for the elliptic cryptosystems designed from CM curves, we may need only to consider the lifting attacks which lift the elliptic curves over finite fields to their Deuring's liftings over number fields and solve the discrete logarithm problems over number fields. However, it is well known that these kind of attacks seem exponentially hard due to difficulty in lifting the rational points of elliptic curves from finite fields to number fields, the exponentially explosion of the heights of these rational points over number fields, and the finite rank of their Mordell-Weil groups. In fact, these are the same arguments known for security of general elliptic cryptosystems [22], i.e., on the immunity of generic elliptic curves from attacks of the index-calculus algorithm.

Existing algorithms used for construction of CM curves, somewhat vaguely called "CM field algorithms", are based on construction of class fields using analytic series expansions of the modular functions over $\boldsymbol{C}$ ([1] and also [7][12])

Although theoretically these algorithms can build any elliptic curve over finite fields, they seemed tedious and always involved with problems such as approximation errors. Their complexity is known as an exponential function $O\left(2^{5 h / 2} h^{21 / 4}\right)$ of the class number $h$, assuming the elliptic curves have their endomorphism rings as an imaginary quadratic order with the class number $h$. Therefore, they becomes impractical for large class numbers. 
Besides, the scenario of the CM field algorithms, i.e. to start from a particular order of an elliptic curve over a given finite field, then calculate the $j$-invariant of the elliptic curve over the class field and finally define the model of the elliptic curve over the finite field with the assigned order, seems somewhat misleading and unnecessarily involved.

In fact, a clearer and simpler scenario consists of two stages. The first one is to build an explicit model of a CM curve over the class field. The second stage is to design the order of the curve over a finite field. The computation time of the first stage is obviously dominant. As long as one has a model of elliptic curve with CM over a number field, as mentioned before, he can use this curve to design different isogenous classes of secure curves over large finite fields.

It maybe interesting to notice the curves over finite fields with the Frobenius endomorphisms with small traces or the endomorphism rings of large class numbers can be most quickly calculated by the SEA algorithms, while the curves with the endomorphism rings of small class numbers or the Frobenius endomorphisms with large traces are most easily dealt by the algorithms using CM curves but are of most time consuming for the SEA algorithms.

Thus, the key issue in using CM curves to design elliptic cryptosystems is to find models of CM elliptic curves over number fields efficiently, in copious supply, and with endomorphism rings of large discriminants or class numbers.

In [25] 26], we shown probabilistic algorithms to find random CM elliptic and higher genus curves by CM tests, which requires no calculation of $j$-invariants or class equations.

In this paper, we first show the CM test algorithms in a more complete form to find random CM curves. Then we show how to construct ring class fields or the definition fields of the CM curves without explicit construction of class equations, i.e. from ray class fields which can be easily derived using division polynomials. Finally, an efficient deterministic algorithm for lifting the ring class equations from small finite fields is presented to construct CM curves. The complexity of this algorithm is of polynomial time in the class number $h: O\left(h^{7}\right)$. All calculations in these algorithms are simple and easy to implement. Since there is only algebraic manipulations involved, no care is needed about approximation errors control during the calculations.

An interesting generalization of these algorithms is to Jacobian varieties of algebraic curves of higher genera [13] (see also [5]).

\section{CM Tests for Elliptic Curves}

We show in this section fast algorithms to test if an elliptic curve over a number field is with $\mathrm{CM}$, which can be used to find random $\mathrm{CM}$ curves over number fields without calculation of $j$-invariants and their class equations.

Definition 1. An elliptic curve $E$ over a field $F$ is with complex multiplication or a CM elliptic curve if its endomorphism ring End $E$ contains the rational integral ring $\boldsymbol{Z}$ as a proper subring. 
We refer the details of theory of complex multiplication to standard references. e.g. 17 32] 18 31.

Definition 2. An elliptic curve which passed a CM test is called a pseudo-CM elliptic curve.

Let $F$ be a number field. Bellow, we denote the residue field of a rational prime $p$ in $F$ as $\boldsymbol{F}_{q}, D$ the discriminant of

an imaginary quadratic number field $\boldsymbol{Q}(\sqrt{\Delta}), \Delta<0$, as CM fields of ordinary elliptic curves, i.e. $\operatorname{End}^{\circ} E:=\operatorname{End} E \otimes_{\boldsymbol{Z}} \boldsymbol{Q}=\boldsymbol{Q}(\sqrt{\Delta})$.

\section{Algorithm 1 (CM tests)}

\section{Procedure 1 (Ordinary reduction) 25]}

Input : Random elliptic curves $E / F$;

Output : Pseudo-CM curves and the discriminants of their CM fields.

Step 1 Choose a small prime $p_{1}$ such that $E / \boldsymbol{F}_{q_{1}}$ is an ordinary reduction. Find the discriminant $d_{1}=l_{1}^{2} \Delta_{1}\left(\Delta_{1}\right.$ : square free) of the characteristic polynomial of the Frobenius endomorphism;

Step 2 Choose small primes $p_{i}, i=2, \cdots, N$ such that $\left(\frac{\Delta_{1}}{p_{i}}\right)=1$, then for $E / \boldsymbol{F}_{q_{i}}$ find the discriminant of the Frobenius endomorphism $d_{i}=l_{i}^{2} \Delta_{i}$. If $\Delta_{i}=$ $\Delta_{1}$ for all $i$, output $E$ as a pseudo-CM curve with the discriminant $D_{1}$. Otherwise, output $E / F$ as without CM.

\section{Procedure 2 (Supersingular reduction)}

Input : Random elliptic curves $E / F$;

Output : Pseudo-CM curves and the discriminants of their CM fields.

Step 1 Choose a small prime $p_{1}$ such that $E / \boldsymbol{F}_{q_{1}}$ is an ordinary reduction. Find the discriminant $d_{1}=l_{1}^{2} \Delta_{1}$ of the characteristic polynomial of the Frobenius endomorphism;

Step 2 Choose small primes $p_{i}, i=2, \cdots, N$ such that $\left(\frac{\Delta_{1}}{p_{i}}\right)=-1$, then if $E / \boldsymbol{F}_{p_{i}^{2}}$ are supersingular or an additive bad reduction for all $i$, output $E$ as a pseudo-CM curve with the discriminant $D_{1}$. Otherwise, output $E / F$ as without CM.

\section{Procedure 3 (bad reduction)}

Input : Random elliptic curves $E / F$;

Output : Pseudo-CM curves and the discriminants of their CM fields.

Step 1 Choose a small prime $p_{1}$ such that $E / \boldsymbol{F}_{q_{1}}$ is an ordinary reduction. Find the discriminant $d_{1}=l_{1}^{2} \Delta_{1}$ of the characteristic polynomial of the Frobenius endomorphism;

Step 2 Choose small primes $p_{i}$ such that $\left(\frac{\Delta_{1}}{p_{i}}\right)=0$, then if $E / \boldsymbol{F}_{p_{i}^{2}}$ are supersingular or an additive bad reduction for all $i$, output $E$ as a pseudo-CM curve with the discriminant $D_{1}$. Otherwise, output $E / F$ as without CM. 
Remark 1: The calculations in these tests can be obviously done by fast algorithms in polynomial time in $\log p_{a m x}$.

Remark 2: With extra computations, the step 2 can be refined to identify the isomorphism types of the endomorphism rings by e.g. Kohel's algorithm [15]. In that case, one will be able to output the discriminants of the endomorphism rings.

Remark 3: Naturally one can combine these procedures to raise the computational efficiency. e.g. after the step 1 , for the first $N$ primes apply the step 2 of each procedures. Besides, the latter two ones should be applied first. Ordinary reductions over prime fields are also preferable.

If the class equation was known, one can use the following test which is based on the result of Gross-Zagier [11], which is very sharp but the calculation of discriminant becomes heavy for large class numbers.

\section{Gross-Zagier test}

Input $f(x) \in \boldsymbol{Z}[x]$ : a polynomial; $d \in \boldsymbol{Z}_{-}$: discriminant of a CM field. Output If $f(x)$ is the class polynomial $H_{d}(x)$.

Step 1 If the constant term of $f(x)$ is not $d$-smooth, output NO;

Step 2 If the discriminant of $f(x)$ is not $(3 / 4) d$-smooth, output NO;

Step 3 Output $f(x)=H_{d}(x)$.

\section{Construction of Ring Class Fields from Ray Class Fields}

Let $k=\boldsymbol{Q}(\sqrt{\Delta})(\Delta<0$ : square free) be an imaginary quadratic number field, $D$ the discriminant of $k, h(D)$ the class number of $k, \mathcal{O}_{k}$ the integral ring of $k, \mathcal{O}_{c}$ an order of $\mathcal{O}_{k}$ of conductor $c$ with discriminant $d=c^{2} D, k_{\text {ring }}^{c}$ the ring class field modulo $(c), k_{\text {ray }}^{c}$ the ray class field modulo $(c), k_{a b s}$ the abstract or Hilbert class field of $k . H_{D}(x)$ the Hilbert class equation and $H_{d}(x)$ the ring class equation of $\mathcal{O}_{c}$.

Let $F$ be a number field, an elliptic curve $E / F$ is with $\mathrm{CM}$ if its endomorphism ring $\operatorname{End}_{F} E$ is an order $\mathcal{O}_{c}$ of an imaginary quadratic number field $k=\boldsymbol{Q}(\sqrt{\Delta})$, where $c$ is the conductor of $\mathcal{O}_{c}$. Furthermore, an elliptic curve with CM has a model over the ring class field modulo $(c), k_{\text {ring }}^{c}$. (Specifically, certain subfields sometimes called its moduli fields.)

In fact, to construct a ring class field, over which the CM elliptic curves are defined, is not the same thing to find the singular moduli or the $j$-invariants which generate the ring class field. As shown bellow, the former could be much easier than the latter.

It is known that one can obtain ring class fields from the genus fields when $d$ are chosen as Euler's convenient numbers. Bellow, we obtain the ring class fields from the ray class fields easily. Let $x_{c}$ be the $x$-coordinate of a $c$-torsion point in $E[c], w_{D}=\frac{D+\sqrt{D}}{2}, h\left(w_{D}, \mathcal{O}_{c}\right)$ the Weber function. 


\section{Algorithm 2}

Input : $d=c^{2} D$ : Discriminant of an imaginary quadratic order in $k=\boldsymbol{Q}(\sqrt{\Delta})$ or $c$ : its conductor;

Output : A moduli field $\boldsymbol{Q}\left(j\left(\mathcal{O}_{c}\right)\right)$ of a ray (ring) class field modulo $(c)$. Step 1 Find an elliptic curve $E / k_{a b s}$ with CM such that

$$
\operatorname{End}_{k_{a b s}} E=\mathcal{O}_{k}
$$

whose $j$-invariant equals $j\left(\mathcal{O}_{k}\right) \in k_{a b s}$;

Step 2 Calculate the $c$-division polynomial of $E, \varphi_{c}(x) \in \boldsymbol{Z}[x]$;

Step 3 Find irreducible factors of $\varphi_{c}(x), f_{c}(x)$;

Step 4 Find the moduli fields in $k_{\text {ray }}^{c}: \boldsymbol{Q}\left(j\left(\mathcal{O}_{k}\right), x_{c}\right)=\boldsymbol{Q}\left(j\left(\mathcal{O}_{c}\right), h\left(w_{D}, \mathcal{O}_{c}\right)\right)$ which are generated by twice simple extensions with the $H_{D}(x)$ and $f_{c}(x)$ as the minimal polynomials.

Remark 1: Since the division polynomials can be easily calculated, one may wish to chose $k=\boldsymbol{Q}(\sqrt{\Delta})$ with small class number $h(D)$.

Remark 2: If one chooses $h(D)=1$, or one uses the thirteen elliptic curves over $\boldsymbol{Q}$, then one finds in $k_{\text {ray }}^{c}$ the moduli field $\boldsymbol{Q}\left(x_{c}\right)=\boldsymbol{Q}\left(j\left(\mathcal{O}_{c}\right), h\right)$ with the minimal polynomial as $f_{c}(x)$.

In this way, one can readily produce a ring class field or its moduli field, which can be used in the CM test algorithms in the previous section as definition fields of CM elliptic curves.

However, since CM curves over a particular number field are of finite number so the probability to find them could be very low. One may wish to use more efficient and deterministic algorithm to find a CM curve by calculation of the ring class equation $H_{d}(x)$, which is also a minimal polynomial of $\boldsymbol{Q}\left(j\left(\mathcal{O}_{c}\right)\right)$. To present such an algorithm will be the task of the following section.

\section{Lifting CM Elliptic Curves fROM Finite Fields}

Let $E(j)$ as a model of an elliptic curve with $j$ as its $j$-invariant . For $p \neq 2,3$,

$$
E(j): \quad y^{2}=x^{3}-\frac{3 j}{j-1728} x-\frac{2 j}{j-1728}
$$

or for any characteristics,

$$
E(j): \quad y^{2}+x y=x^{3}-\frac{36}{j-1728} x-\frac{1}{j-1728} .
$$

\section{Algorithm 3:}

Input : $d=c^{2} D<0$ : Discriminant of an imaginary quadratic order in $\boldsymbol{Q}(\sqrt{\Delta})$; Output : The ring class equation $H_{d}(x)$. 
step 1 Using the Algorithm 2 to construct a moduli field $F$ of a ring class field $k_{\text {ring }}^{c}$;

step 2 For small prime $p_{i}$, let $\boldsymbol{F}_{q_{i}}$ be the residue field of the rational prime $p_{i}$ in $\mathcal{O}_{F}$.

1. If $\left(d / p_{i}\right)=1$, find among all $E(j) / \boldsymbol{F}_{q_{i}}$ for $\forall j \in \boldsymbol{F}_{q_{i}} h(d)$ isogenous but non-isomorphic elliptic curves $E_{s}, s=1, \cdots, h(d)$ such that

$$
4 q_{i}=t_{i}^{2}-c_{i}^{2} d, \quad \text { where } t_{i}=q_{i}+1-\# E_{s}\left(\boldsymbol{F}_{q_{i}}\right)
$$

Then from the $h(d) j$-invariants of $E_{s}, j_{i s} \in \boldsymbol{F}_{q_{i}}$, calculate the ring class equation $\bmod p_{i}$ as

$$
H_{d}(X) \bmod p_{i} \equiv \prod_{s=1}^{h(d)}\left(x-j_{i s}\right)
$$

2. If $\left(d / p_{i}\right)=-1$, find among all $E(j) / \boldsymbol{F}_{p_{i}^{2}}$ for $\forall j \in \boldsymbol{F}_{p_{i}^{2}}, h(d)$ isogenous but non-isomorphic elliptic curves $E_{s}, s=1, \cdots, h(d)$ s.t. they are supersingular or additive bad reductions.

Then from $h(d) j$-invariants of $E_{s}, j_{i s} \in \boldsymbol{F}_{p_{i}^{2}}$ calculate the ring class equation $\bmod p_{i}$

$$
H_{d}(X) \bmod p_{i} \equiv \prod_{s=1}^{h(d)}\left(x-j_{i s}\right)
$$

3. If $\left(d / p_{i}\right)=0$, find among all $E(j) / \boldsymbol{F}_{p_{i}^{2}}, \forall j \in \boldsymbol{F}_{p_{i}^{2}}, h(d)$ isogenous but non-isomorphic elliptic curves $E_{s}$ s.t. $E_{s} / \boldsymbol{F}_{p_{i}^{2}}$ are supersingular or additive bad reductions.

Then from $h(d) j$-invariants of $E_{s}, j_{i s} \in \boldsymbol{F}_{p_{i}^{2}}$ calculate the ring class equation $\bmod p_{i}$

$$
H_{d}(X) \bmod p_{i} \equiv \prod_{s=1}^{h(d)}\left(x-j_{i s}\right)
$$

step 3 By the Chinese Remainder Theorem (CRT) to lift the coefficients of

$$
H_{d}(x) \bmod \prod_{i} p_{i}
$$

to $Z[x]$

Step 4 If the $E(j)$ defined by the lifted ring class equation passes the CM tests in the Algorithm 1 or the Gross-Zagier test, then output $H_{d}(x) \bmod \prod_{i} p_{i}$ as the ring class equation $H_{d}(x)$, and $E(j)$ as a pseudo-CM elliptic curve; If not goto step 3 to add one more prime $p_{i}$ or try other combinations.

Remark 1: Considering increase of the size of the coefficients of class equations, one may first calculate the Weber class invariants $f$ from $j$-invariants over finite fields,

$$
(x-16)^{3} \equiv j x \bmod p_{i}, \quad x \equiv f^{24} \bmod p_{i}
$$


then lift the Weber class equations, which will allow us to lift the class equations of large class numbers. The details are referred to 7] [1] etc.

Remark 2: To avoid the combinations, one can use only $p$ s.t. there is few ambiguity in conductors. A more complete version should include the Kohel's deterministic algorithm to distinguish the isomorphism types of full endomorphism rings of the elliptic curves $E_{s} / \boldsymbol{F}_{q_{i}}[15$. e.g. in ordinary lifting to choose $E_{s}$ s.t.

$$
\text { End }_{\boldsymbol{F}_{q_{i}}} E_{s}=\mathcal{O}_{c}=\boldsymbol{Z}+c \mathcal{O}_{k}
$$

In supersingular lifting, check at first that if the endomorphism rings of the curves contain an optimal embedding of $\mathcal{O}_{c}$, the imaginary order which is chosen as the endomorphism ring of the target CM curve.

Remark 3: In fact, lifting from only prime fields is possible once a discriminant is chosen appropriately. Thus there is no need to build the ring class field a priori even in implicit form. Thus, by using only the lifting from prime fields, calculation of the division polynomials can also be omitted. In fact, the Hilbert class equations can also be lifted in the similar way.

\section{Examples of CM Tests}

We applied the ordinary reduction CM test of the Algorithm 1 to the elliptic curve over $Q$ :

$$
y^{2}=x^{3}-\frac{3 \cdot j}{j-1728} x-\frac{2 \cdot j}{j-1728}
$$

and take $p_{1}=709$, the other small primes $p_{i}>300(i=2, \cdots, 10)$.

The nine tests $(i=2, \ldots, 10)$ for 64847 random curves are shown in the table, the second row shows the number of non-CM curves which are rejected at the $i$-th tests in Step 2. It can be observed that almost all of them are rejected by double or triple tests and none of the non-CM curves passed the first five tests.

\begin{tabular}{|c|c|c|c|c|c|c|c|c|c|}
\hline$i$-th test & 2 & 3 & 4 & 5 & 6 & 7 & 8 & 9 & 10 \\
\hline$\#\{$ rejected curves $\}$ & 64301 & 518 & 25 & 0 & 1 & 0 & 0 & 0 & 0 \\
\hline
\end{tabular}

There are only two curves which passed the fifth and all tests, i.e. $D_{1}=D_{i},(i=$ $2, \cdots, 10)$. Their j-invariants are

$$
j=-15^{3},-32^{3} .
$$

As we know, they are truly CM curves.

In fact, the supersingular and bad reduction tests distinguish even sharperly between CM and non-CM curves (see proof of the algorithm 1 for reasons).

\section{Examples of Construction of CM Curves}

Take $h=174, d=-153164$ with $(c=118, D=-11)$. The Weber class polynomial $W_{d}(x)$ is lifted from the following 19 ordinary reduction over prime fields.

$$
p_{i} \in\{38327,38867,39191,47507,51287,52691,54167,62627,68567,
$$
79907, 84947, 93047, 95891, 98807, 111191, 114467, 128291, 131927, 167891\} 
Bellow, we show a part of it with small coefficients.

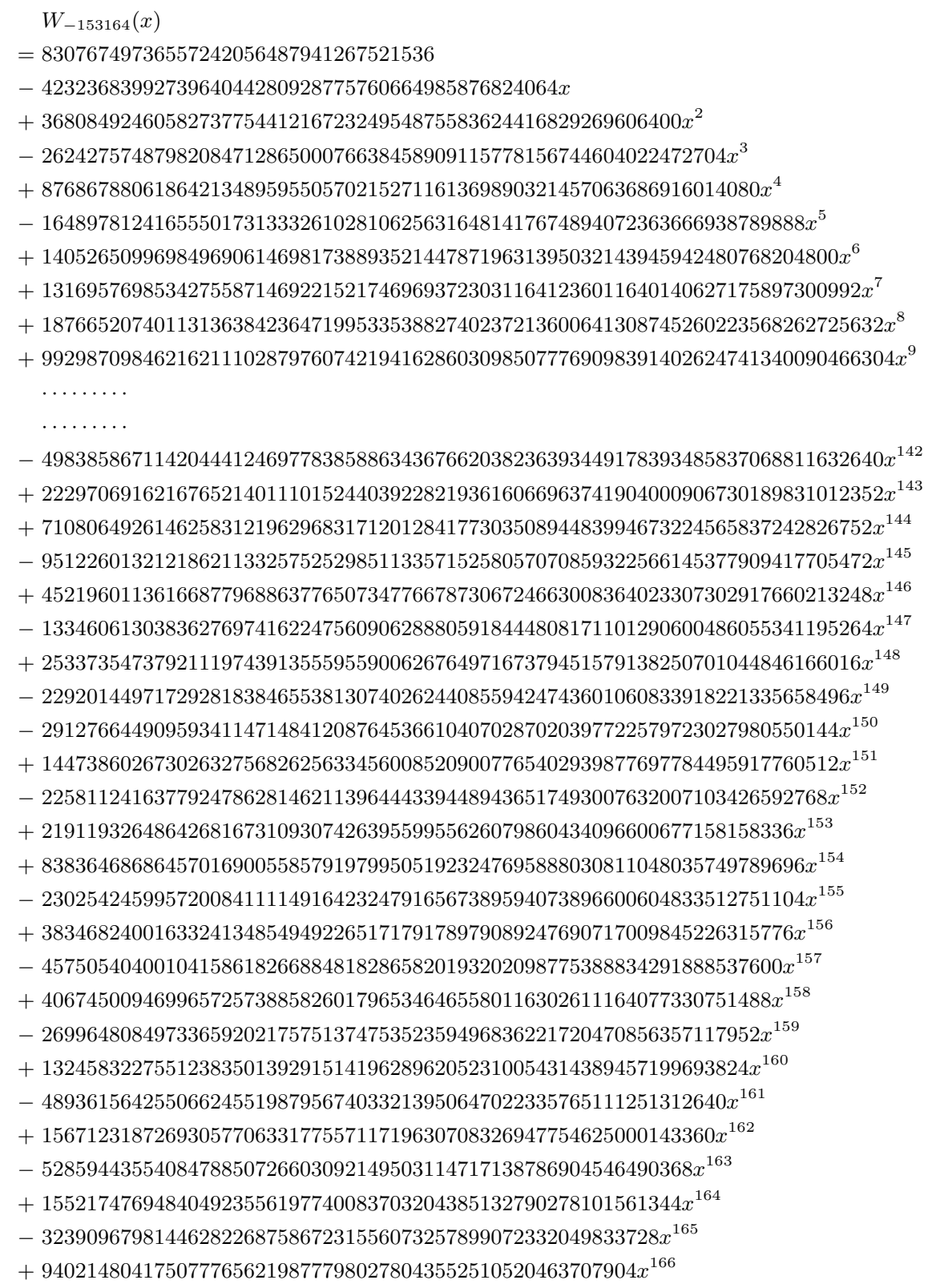


- $229630960711252290445040913713427951765596468574464 x^{167}$

$-3429592761776584363504935760776221071566271657712 x^{168}$

- $9752861942166664038528762369130374074251013536 x^{169}$

$-553178966475435712930872308777998684975056 x^{170}$

$-12039696790209383877109384281205985464 x^{171}$

$+159660091018550701603559721992 x^{172}$

$-17722643006531637142016 x^{173}+x^{174}$

The discriminant of the class polynomial and its constant term passed the GrossZagier test, the latter has factorization as

$$
83076749736557242056487941267521536=2^{116}
$$

\section{Examples for Cryptosystem Design}

Use the above CM curve $(d=-153164)$, an almost prime elliptic curve $E$ over a prime finite field $\boldsymbol{F}_{p}$ is obtained using the Algorithm 4 in the Appendix, where

$$
\begin{aligned}
p & =6411233586778658698012854170834647184757484423031 \\
\# E\left(\boldsymbol{F}_{p}\right) & =6411233586778658698012849108768570758807340538364 \\
& =2^{2} * 3 * 1039 * p_{\max } \\
p_{\max } & =514215077540797136510494795377652450979093723
\end{aligned}
$$

The curve has its $j$-invariant as

$$
j-\text { inv }=37794183507581776432669939667057046706278554144
$$

The definition equation of this curve $y^{2}=x^{3}+a x+b$ has the following coefficients.

$$
\begin{aligned}
& a=4424050837045189024624780453466068541773776795332 \\
& b=5086445086956345582420805025922261422768346004565
\end{aligned}
$$

\section{Proof of the Algorithms}

Theorem 3. In the Algorithm 1 ,

(1) Any elliptic curve $E / F$ rejected is without complex multiplication;

(2) The elliptic curves accepted are with high probability with complex multiplication.

Sketch of proof: Without loss of generality, we assume that $F \supset k$. (If not one can use $F:=F k$ ).

According to the Néron-Ogg-Shafarevitch criterion, the Grossencharacter $\psi_{E / F}$ is unramified if and only if the elliptic curve $E / F$ has good reduction. In particular, for CM curves, if $p_{i}$ splits in $\boldsymbol{Q}(\sqrt{\Delta}), E / \boldsymbol{F}_{q_{i}}$ is an ordinary reduction. While 
only when the $p_{i}$ is inert or ramified in $\boldsymbol{Q}(\sqrt{\Delta}), E / \boldsymbol{F}_{q_{i}}$ could have supersingular good reduction.

It is known by a result of Serre and Tate that CM curves have potential good reduction. Thus they only could have unstable or additive bad reduction.

Let $\mathfrak{P}_{i}$ be a prime ideal of $\mathcal{O}_{F}$ lying over $p_{i}$. By the Deuring's reduction theory of elliptic curves, the reduction mod $\mathfrak{P}_{i}$ induces an injective ring homomorphism of endomorphisms of the elliptic curve $\operatorname{End}_{F} E$ to End $\boldsymbol{F}_{q_{i}} E$. This map however induces a field isomorphism between the endomorphism fields if the elliptic curve is with $\mathrm{CM}$ and the reduction is ordinary.

Thus, if a curve $E$ is non-CM and $E / \boldsymbol{F}_{q_{i}}$ is an ordinary reduction, then End ${ }_{\boldsymbol{F}_{q_{i}}} E$ will be imaginary quadratic fields with random discriminants. On the other hand, if a curve is CM, one will have the same $\operatorname{End}_{\boldsymbol{F}_{q}} E$ for any ordinary reduction at $p$. Thus, the ordinary test will always reject non-CM curves and after $N$ repetance of the ordinary reduction, the survived curves will be with $\mathrm{CM}$ in probability larger than $1-1 / 2^{N}$.

As to the supersingular reduction, it is known by Serre that for generic curves, the set of primes $S(x)=\left\{p_{i}<x \mid E / \boldsymbol{F}_{q_{i}}\right.$ is supersingular $\}$ has density of zero and is conjectured that $\# S(x) / \pi(x)=O(1 / \sqrt{x})$. The bad reduction at the chosen primes is even much rare for non-CM curves. Therefore, the later two tests can abandon non-CM curves even more efficiently or with higher probability. $Q E D$

Theorem 4. The Algorithm 2 outputs the moduli field $\boldsymbol{Q}\left(j\left(\mathcal{O}_{c}\right)\right)$ of the ring class field $k_{\text {ring }}^{c}$ modulo $(c)$.

Sketch of proof: By the second main theorem of complex multiplication[17] [32] [31],

$$
k_{\text {ray }}^{c}=k_{a b s}\left(x_{c}\right)=k\left(j\left(\mathcal{O}_{k}\right), x_{c}\right) .
$$

where $x_{c}$ is the $x$-coordinate of a $c$-torsion point in $E[c], k_{\text {ray }}^{c}$ and $k_{\text {ring }}^{c}$ are the ray class field and the ring class field modulo $(c), k_{a b s}=k\left(j\left(\mathcal{O}_{k}\right)\right)$ and $k_{\text {ring }}^{c}=k\left(j\left(\mathcal{O}_{c}\right)\right)$.

On the other hand, one knows that

$$
k_{\text {ray }}^{c}=k_{\text {ring }}^{c}\left(h\left(w_{D}, \mathcal{O}_{c}\right)\right)=k\left(j\left(\mathcal{O}_{c}\right), h\left(w_{D}, \mathcal{O}_{c}\right)\right)
$$

where $w_{D}=\frac{D+\sqrt{D}}{2}, h\left(w_{D}, \mathcal{O}_{c}\right)$ is the Weber function.

Thus, the ring class field $k_{\text {ring }}^{c}$, as an extension of $k_{a b s}$ also, can be constructed as a subfield of the ray class field $k_{\text {ray }}^{c}$. Its moduli field can be found also as a subfield.

$Q E D$

Theorem 5. The Algorithm 3 outputs the ring class equation $H_{d}(x)$, thus all the $C M$ curves $E$ with the End $E=\mathcal{O}_{c} \subset \mathcal{O}_{k}$, where $k=\boldsymbol{Q}(\sqrt{\Delta})$.

Sketch of proof: Here we assume that the imaginary quadratic field $k$ is not contained in the moduli field $F=\boldsymbol{Q}\left(j_{c}\right)$ of $k_{\text {ring }}^{c}$. Then $K=k_{\text {ring }}^{c}=F k$. A rational prime $p \in \boldsymbol{Z}$ decomposes into $(p) \mathcal{O}_{k}=\mathfrak{p p}$ ' where $\mathfrak{p}$. $\mathfrak{p}$ ' are prime ideals in $\mathcal{O}_{k}$. Similarly, a prime ideal $\mathfrak{P}$ in $\mathcal{O}_{F}$ decomposes into $\mathfrak{P} \mathcal{O}_{K}=\mathfrak{Q Q}$ '. 
By the criterion of Néron-Ogg-Shararevitch, one can prove that $E$ has everywhere potentially good reduction which means that $j$ is integral at all primes $\mathfrak{Q}$ in $\mathcal{O}_{K}$. Thus for CM curves, the minimal polynomials of $j H_{d}(x) \in \boldsymbol{Z}[x]$, one can find the ring class equation $H_{d}(x) \bmod p_{i}$ from $E_{s i} \bmod \mathfrak{Q}_{i}$ where $\mathfrak{Q}_{i}$ lying over $p_{i}$ then use the CRT to recover the $H_{d}(x)$.

The next task is to look for reduction of $h(d) j$-invariants of CM elliptic curves which are Galois conjugates each other. This can be done using again ramificiation properties of the Grossencharacter $\psi_{E / K}$.

e.g. the EmodP is a good reduction if and only if $\psi_{E / K}$ is unramified. Particularly Emod $\mathfrak{P}$ is ordinary reduction if and only if $\mathfrak{P}$ splits in $\mathcal{O}_{K}$ or $\mathfrak{p} \neq \mathfrak{p}$ ' or $(d / p)=1$. EmodP is a supersingular reduction or additive bad reduction if $\mathfrak{P}$ remains inert or ramifies in $\mathcal{O}_{K}$, i.e., $p$ remains inert or ramifies in $\mathcal{O}_{k}$ or $(d / p)=0,-1$.

These features can be distinguished from the endomorphism rings or calculation with the Frobenius endomorphism.

Besides, it is easy to prove that the degrees of $p_{i}$ 's which induce supersingular and bad reductions in the moduli field $\boldsymbol{Q}\left(j\left(\mathcal{O}_{c}\right)\right)$ are less than two. Thus, it is enough to look for the candidates of CM $j$-invariants over $\boldsymbol{F}_{p_{i}^{2}}$.

$Q E D$

\section{Complexity Analysis}

It is known that for the CM field algorithm by Atkin and Morain, the precision needed to calculate a class equation with the class number $h$ is by 1 ]

$$
\operatorname{Prec}(d)=\left(\begin{array}{c}
h \\
\frac{h}{2}
\end{array}\right) \frac{\pi \sqrt{d}}{\log 10} \sum \frac{1}{a}+v_{0}
$$

The number of terms required in series expansion of $j$-invariants is

$$
\sqrt{S a / k} \text { where } S=\frac{2(\log 6+\operatorname{Prec}(d) \log 10)}{3 \pi \sqrt{d}}
$$

Using Sterling's formula: $n !=(n / e)^{n} \sqrt{2 \pi n}$, and $h=O(\sqrt{d}), \operatorname{Prec}(d)=O\left(2^{h} h^{3 / 2}\right)$ and the number of terms required in series expansion is $O\left(2^{h / 2} h^{1 / 4}\right)$. Thus its complexity is of an exponential function of $h$.

Now we analyze the complexity of the Algorithm 3. Since $j=O\left(e^{\pi \sqrt{d}}\right)$, the largest coefficient in the class equations is in order of $j^{h}=O\left(e^{\pi h \sqrt{d}}\right)=O\left(e^{\pi h^{2}}\right)$. To lift it by the CRT, it needs to repeat the lifting procedures over $O\left(h^{2}\right)$ finite fields $\boldsymbol{F}_{q_{i}}$, of which the sizes are also $O\left(h^{2}\right)$. Checks through all elements of each $\boldsymbol{F}_{q_{i}}$ as candidates of $j$-invariants of CM curves in step 2 cost $O\left(h^{2}\right)$. If e.g. in ordinary lifting the Kohel's deterministic algorithm is used to identify the isomorphism types of full endomorphism rings of the elliptic curve $E_{s} / \boldsymbol{F}_{q}$, which runs in time $O\left(q^{1 / 3+\epsilon}\right)$ for any $\epsilon>0\left[15\right.$, then step 2 will cost $O\left(q_{i}^{4 / 3+\epsilon}\right)$ for each $\boldsymbol{F}_{q_{i}}$, in all $O\left(q_{i}^{7 / 3+\epsilon}\right)=O\left(h^{14 / 3+2 \epsilon}\right)$. On the other hand, the calculations in step 3 to calculate the coefficients of $H_{d}(x)$ by the CRT will be dominant, which costs $O\left(h^{7}\right)$. In conclusion, the whole calculations will be in complexity of $O\left(h^{7}\right)$. 
Acknowledgment: The authors wish to thank Prof. Yasutaka Ihara for kind suggestion of [11, Prof. Fumiyuki Momose for helpful discussions, and Mr. Kazuto Matsuo for assistance in preparation of this paper.

\section{Appendix: Design of Secure Elliptic Cryptosystems with CM Curves}

Once we have a CM elliptic curve over a number field, we can use it to design elliptic curve over finite fields using the fast algorithms of reduced quadratic forms(see appendix) or Cornnachia's algorithm[4] 25]. Bellow, we show an algorithm using reduced quadratic forms.

\section{Algorithm 4}

Input $E / F$ : A CM elliptic curve; $d$ : the discriminant of its endomorphism ring. Output $q$ such that $E / \boldsymbol{F}_{q}$ is an almost prime curve.

Step 1 Choose $q=p^{n}($ If $p=2$ assume $d \equiv 1 \quad(\bmod 8))$;

Step 2 Find an $m_{0}$ such that $m_{0}^{2} \equiv d \quad(\bmod 4 q)$;

Step 3 Let $n^{\prime}=4 q, m^{\prime}=2 m_{0}, l^{\prime}=\left(m_{0}^{2}-d\right) / 4 q$. If the reduced binary form of $g\left(x^{\prime}, y^{\prime}\right)=n^{\prime} x^{2}+m^{\prime} x^{\prime} y^{\prime}+l^{\prime} y^{\prime 2}$ is not $f(x, y)=x^{2}-4 d y^{2}$, go to step 2 ;

Step 4 Calculate the modular transform $A$ from $g\left(x^{\prime}, y^{\prime}\right)$ to $f(x, y)$ such that

$$
\left[\begin{array}{l}
x \\
y
\end{array}\right]=A\left[\begin{array}{l}
x^{\prime} \\
y^{\prime}
\end{array}\right], \quad A=\left[\begin{array}{ll}
a_{11} & a_{12} \\
a_{21} & a_{22}
\end{array}\right]
$$

Step 5 Let $t=a_{11}$, check if $\# E\left(\boldsymbol{F}_{q}\right)=q+1-t$ contains a large prime factor, or almost prime. If not, go to step 2 to find a new $m_{0}$ or go to step 1.

\section{References}

1. A.O.L.Atkin, F. Morain : "Elliptic Curves and Primality Proving" , Research Report 1256, INRIA, Juin (1990).

2. J. Chao, K. Tanada, S. Tsujii : "On secure elliptic curves against reduction attack and their design strategy", IEICE, Symposium on Cryptography and Information Security, SCIS'94, 10A, 1994-1. IEICE, Tech. rep. ISEC-93-100, p29-37, Mar. (1994)

3. J. Chao, K. Tanada, S. Tsujii : "Design of Elliptic Curves with Controllable Lower boundary of Extension Degree for Reduction Attacks", Yvo G. Desmedt (Ed.) Advances in Cryptology-CRYPTO'94, Lecture Notes in Computer Science, 839, Springer-Verlag, pp.50-55, (1994)

4. J. Chao, K. Harada, N. Matsuda, S. Tsujii : "Design of secure elliptic curves over extension fields with CM fields methods", IEICE, Symposium on Cryptography and Information Security, SCIS'94, A5.5, 1995-1. IEICE, Tech. rep. ISEC-95-52, p.1-12, 1995-3. Proc. of Pragocrypto'96, p.93-108, (1996)

5. J. Chao, N. Matsuda, S. Tsujii "Efficient construction of secure hyperelliptic discrete logarithm problems" Springer-Verlag Lecture Notes on Computer Science, Vol.1334, pp.292-301, "Information and Communication Security" Y. Han, T. Okamoto, S. Qing (Eds.) Proceedings of First International Conference ICICS'97, Beijing, China, Nov., (1997) 
6. H. Cohen : "A course in computational algebraic number theory", Springer, GTM138, (1995)

7. H. Cohn : "Construction of class fields", Cambridge Univ. Press, 1978.

8. J.-M. Couveignes, F. Morain, "Schoof's algorithm and isogeny cycles", Proceedings of ANTS'I, May, 1994. Lecture Notes in Computer Science, Springer-Verlag, pp.4358. (1994)

9. D. Cox : "Primes of the forms $x^{2}+n y^{2}$ ", John Wiley and Sons. (1989)

10. N. D. Elkies "Elliptic and modular curves over finite fields and related computational issues" "Computational perspectives on number theory", Proceedings of a Conference in Honor of A.O.L. Atkin, AMS, D.A.Buell, and J.T. Teitelbaum ed. pp.21-76, Sept. (1995)

11. B. Gross, D. Zagier : "On singular moduli", J. reine angew. Math. 355, pp.191-220. (1985)

12. E. Kaltofen and N. Yui, "Explicit construction of the Hilbert class fields fo imaginary quadratic fields by integer lattice reduction", New York Number Theory Seminar, 1989-1990, Springer-Verlag, pp. 150-202, (1991)

13. H. Kawasiro, O. Nakamura, J. Chao, S.Tsujii : "Construction of CM hyperelliptic curves using RM families", SCIS'98, 4-1-A, Jan. 1998. IEICE Tech. Rep. ISEC9772, p. 43-50, March, (1998)

14. N.Koblitz : "Elliptic Curve Cryptosystems",Math. Comp.,vol.48, p.203-209, (1987)

15. D. Kohel "Endomorphism rings of ellitpic curves over finite fields" PhD thesis, UCB, (1996)

16. K. Kurotani, K. Matsuo, J. Chao, S. Tsujii : "Consideration of security of hyperelliptic cryptosystems", IEICE, Symposium on Cryptography and Information Security, SCIS'98, 4.1-D, Jan. (1998)

17. S. Lang : "Elliptic Functions", 2nd ed., Springer-Verlag, (1987)

18. S.Lang : "Complex multiplication" Springer-Verlag, (1983)

19. G-J. Lay and H.G. Zimmer : "Constructing Elliptic Curves with Given Group Order over Large Finite Fields", Proceeding of ANTS95, May, (1994)

20. R. Lercier and F. Morain : "Counting the number of points on elliptic curves over finite fields: strategies and performances",Proceeding of EUROCRYPTO'95, (1995)

21. A.Menezes : "Elliptic Curve Public Key Cryptosystems", Kluwer Academic, (1993)

22. V.S.Miller : "Use of Elliptic Curves in Cryptography", Advances in Cryptology Proceedings of Crypto'85, Lecture Notes in Computer Science , 218 , SpringerVerlag, p.417-426, (1986)

23. F. Morain, : "Building cyclic elliptic curves modulo large primes", Advances in Cryptology -EUROCRYPT'91, Lecture Notes in Computer Science. 547 p.328336, (1991)

24. F. Morain, "Calcul du nombre de points sur une courbe elliptique dans un corps fini: aspects algorithmique" Actes des Journees Arithmetiques, (1995)

25. O. Nakamura, N. Matsuda, J. Chao, S. Tsujii : "On cryptosystems based on abelian varieties with CM", IEICE, Symposium on Cryptography and Information Security, SCIS'97, 12-E, 1997-1, IEICE, Tech. Rep. ISEC-96-81, Mar. (1997)

26. O. Nakamura, N. Matsuda, J. Chao, S. Tsujii : "On cryptosystems based on abelian varieties with CM", IEICE, The first symposium on algebraic curves and their applications, Sept. (1997)

27. R. Schoof : "Elliptic curves over finite fields and the computation of square roots $\bmod p$ ", Math. Comp., vol.44, p.483-494, (1985)

28. R. Schoof : "Counting points on elliptic curves over finite fields", Journal de The'orie des Nombres de Bordeaux 7, pp.219-254, (1995) 
29. J.P.Serre, J.Tate : "Good reduction of abelian varieties", Ann. of Math. (2), 88 , p.492-517, (1968)

30. J. H. Silverman : "The Arithmetic of Elliptic Curves", Springer-Verlag, (1988).

31. J. H. Silverman : "Advanced Topics in the Arithmetic of Elliptic Curves", GTM151, Springer-Verlag, (1994)

32. G. Shimura : "Arithmetic theory of automorphic function", Iwanami-Shoten and Princeton, (1971).

33. K. Sobataka, O. Namamura, J. Chao, S.Tsujii : "Construction of secure elliptic cryptosystems using CM tests and Lifting ", SCIS'98, 4-1-B, Jan. (1998). IEICE Tech. Rep. ISEC97-71, p. 35-42, March, (1998).

34. J.Tate : "Endomorphisms of Abelian varieties over finite fields", Invent. Math. 2, p.134-144, (1966) 